\title{
Novel evidence that ApoA-1 deficiency facilitates HSC mobilization and differentiation and halts HSC quiescence and self-renewal, in mice
}

Mastora I'*, Papachristou NI*, Blair HC², Kalyvioti ES', Syggelos SA', Karavia E3, Kypreos KE³, Papachristou D J1,2

'Department of Anatomy-Histology-Embryology, Unit of Bone and Soft Tissue Studies, University of Patras, School of Medicine, Patras, Greece, 2 Department of Pathology, University of Pittsburgh, School of Medicine, Pittsburgh, PA, USA,

\section{${ }^{3}$ Department of Pharmacology, University of Patras, School of Medicine, Patras, Greece}

\section{INTRODUCTION}

Recent evidence suggests that osteoblastic bone marrow niche (BMN) is vital for the maintenance and self-renewal of hematopoietic stem cells (HSC). It has been recently proposed that cholesterol efflux pathways participate in HSC mobilization and that cholesterol-sensing pathways control the proliferation of HSC progenitors. Moreover, we have recently documented that HDL perturbations result in impaired osteoblastic function in mice. In the present study we aimed at investigating the role of ApoA-1, the cardinal regulator of HDL biosynthesis in the regulation of HSC quiescence-mobilization and consequently in hematologic malignancies.

\section{METHODS}

Whole bone marrow cells (WBMCs) were isolated, from the femora of ApoA-1 $(n=6)$ and wild-type (WT) $(n=6)$ C57BL/6 mice and assessed for the expression of factors that are differentially expressed in the BM microenvironment and affect HSC fate. More specifically we tested the expression of the chemoattractant cytokine CLCX12, its receptor CXCR4, the Jagged-1/Notch $(1,2)$ signaling cascade elements as well as $\mathrm{N}$-cadherin and osteopontin, factors that promote HSC quiescence and self-renewal with QRT-PCR. Additionally, we assessed the expression of CXCR4 of HSC with flow cytometry.

\section{RESULTS}

$\operatorname{Re}$

Real time PCR analysis of CXCR4 and CXCL12 in WBMCs devired from apoA-1- and C57BL/6 mice. WBMCs obtained from apoA-1\% mice $(n=4)$ showed a significant increase $(p=0.008)$ in CXCR4 expression and a significant decrease in CXCL12 expression $(p=0.001)$, compared to WT mice $(n=4)$ (Fig.1 ).

Fig. 1
CXCR4

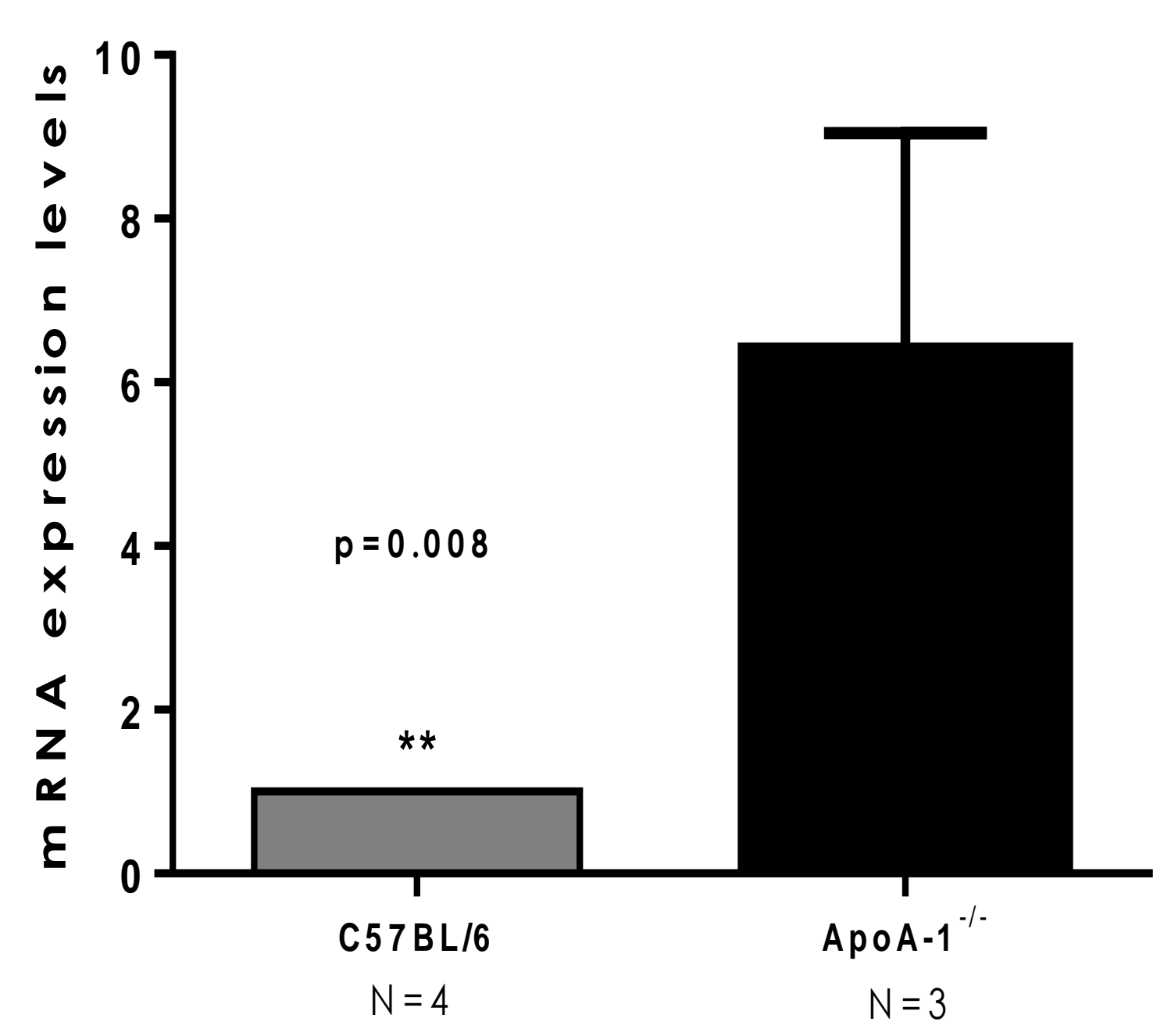

$\mathrm{CXCL12}$

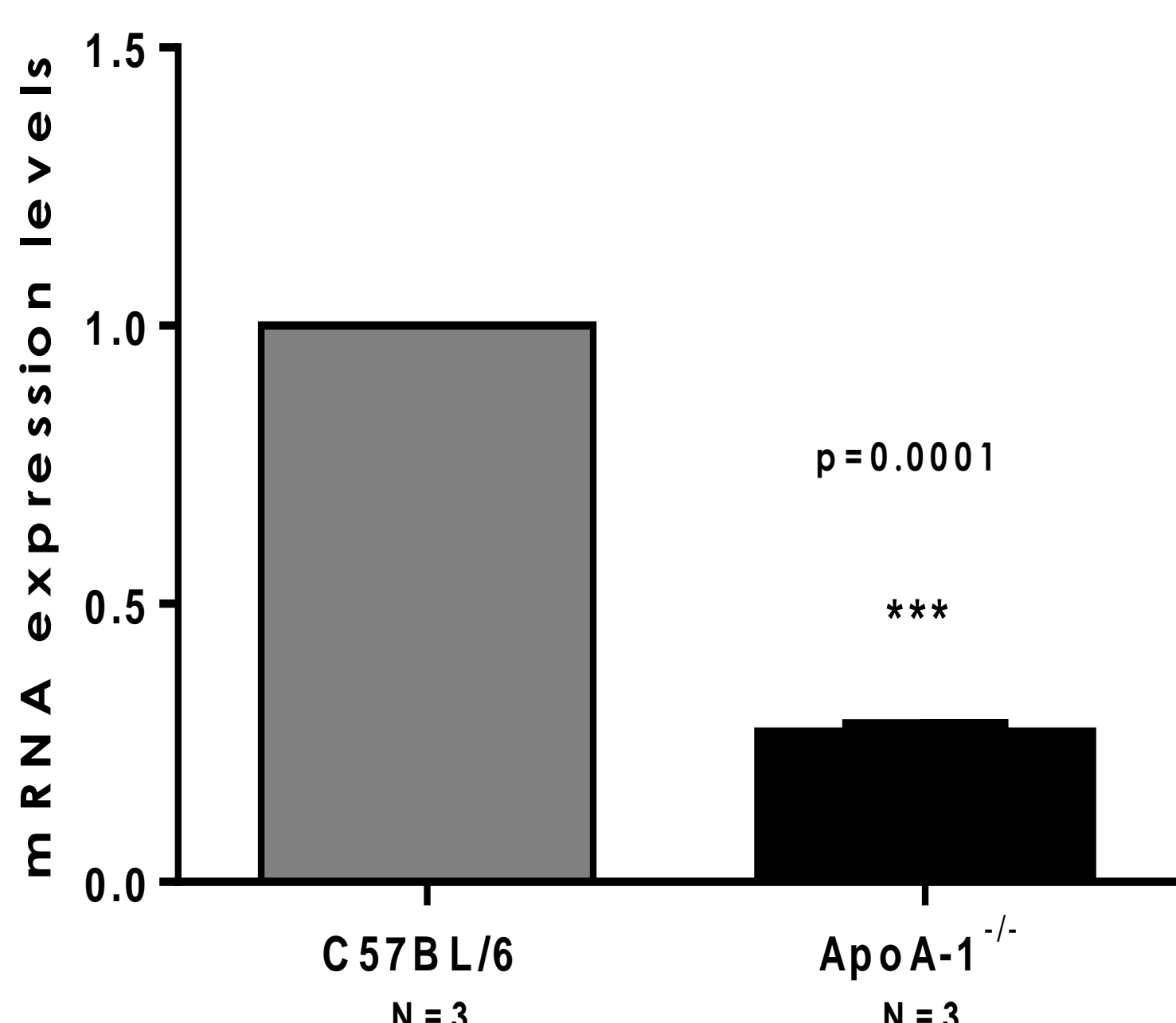

Flow Cytometric analysis of CXCR4 in HSCs derived from apoA-1\% and C57BL/6 mice. The evaluation of CXCR4 protein expression levels on HSCs obtained from apoA $1 \%$ revealed no significant differences compared to C57BL/6 mice (Fig.2 ).

Fig. 2

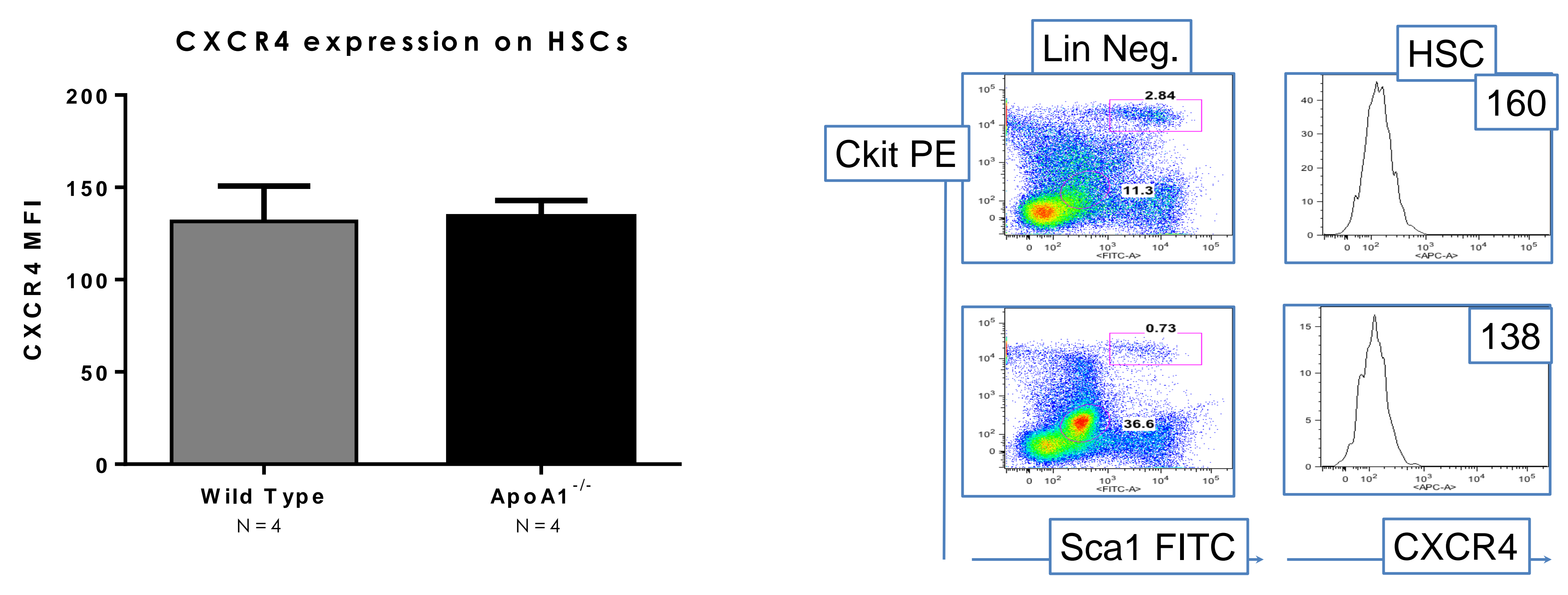

\section{CONCLUDING REMARKS}

\section{SUMMARY OF RESULTS:}

1.The expression of CLCX12 was significantly reduced, while the expression CXCR4 was greatly augmented (possibly via feedback cell reactionmechanism) in the WBMCs of the ApoA-1\% compared to the WT mice.

2.WBMCs from ApoA-1/- mice displayed strongly increased mRNA levels of Jagged-1 compared to the WT mice.

Osteopontin mRNA expression levels were decreased in apoA $1 \%$ mice in contrast to the WT mice.

4.Flow cytometry revealed no significant differences in CXCR4 expression on HSCs of both study groups.

CONCLUSIONS:

The present study suggests for the first time that ApoA-I deficiency (and thus impaired HDL) halts HSC maintenance and quiescence, whereas it promotes HSC differentiation suggesting that it may be involved in the pathobiology of hematologic malignancies and possibly bone metastases.
Real time PCR analysis of the Jagged-1/Notch $(1,2)$ signaling cascade elements in WBMCs devired from apoA-1\% and C57BL/6 mice. Jagged-1 mRNA levels from WBMCs were significantly increased in apoA $1 \%$ mice $(p=0.0163)$, compared to C57BL/6 WT mice. In contrast, Notch $1 \quad(p=0.9705)$ and Notch $2(p=0.1276)$ mRNA levels from bone marrow cells showed no significant differences between both study groups of animals (Fig.3 ).

Fig.3
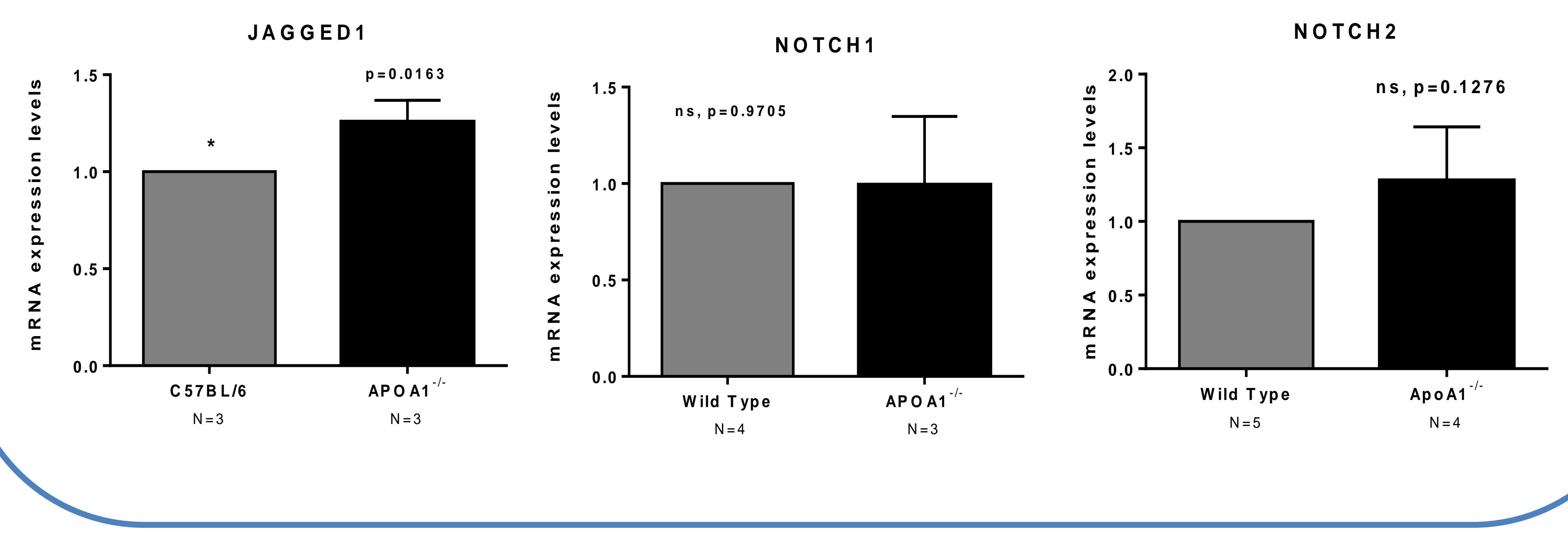

Real time PCR analysis of $\mathrm{N}$-cadherin and Osteopontin in WBMCs devired from apoA $1 \%$ and $\mathrm{C} 57 \mathrm{BL} / 6$ mice. $\mathrm{N}$-cadherin mRNA levels from WBMCs revealed no significant differences between both study groups of animals $(p=0.1278)$ Osteopontin mRNA from bone marrow cells showed a trend to decreased expression in apoA $1 \%$ mice in contrast to WT mice $(p=0.0764)$ (Fig.4).

Fig.4
N-CADHERIN

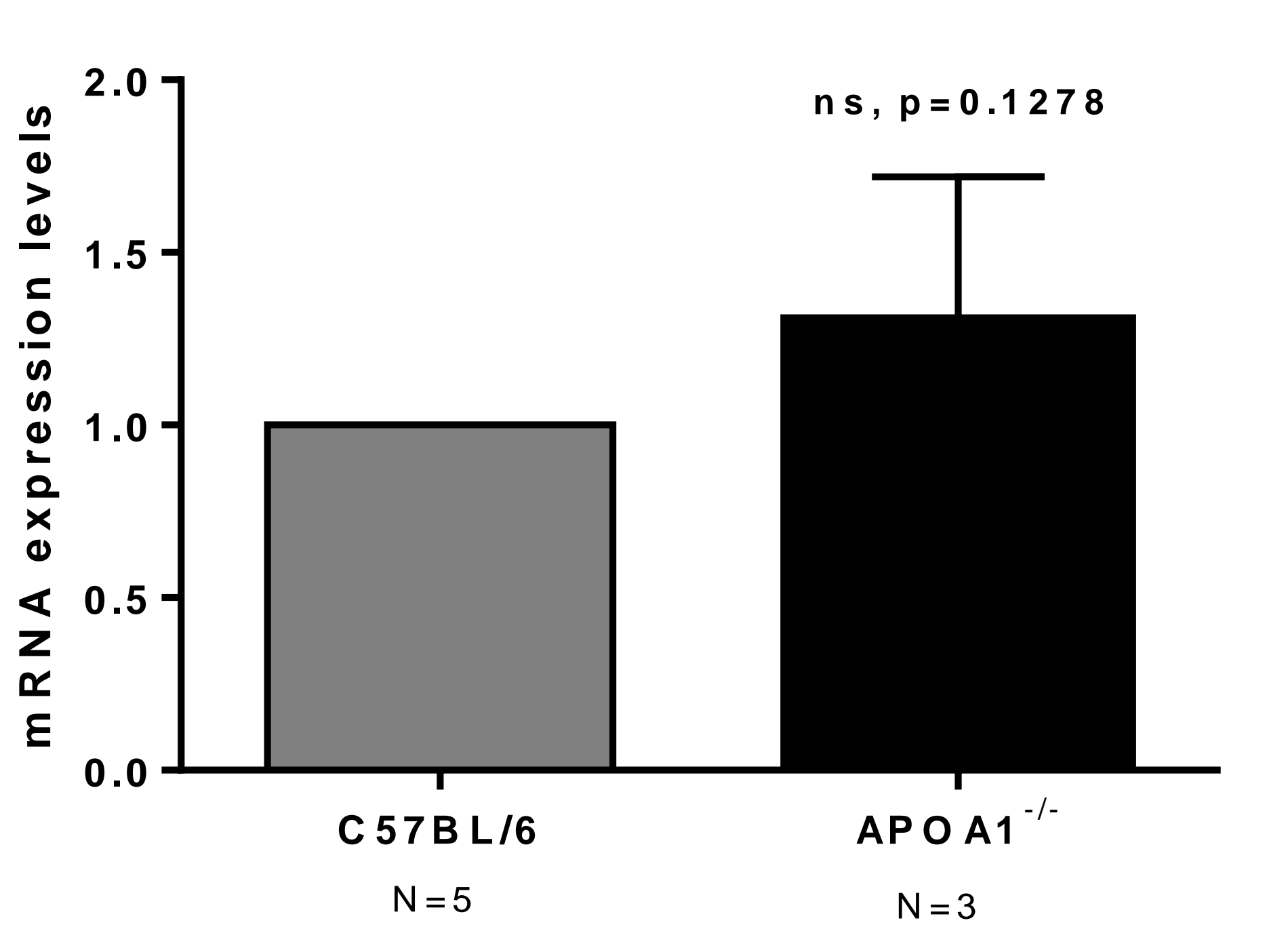

OSTEOPONTIN

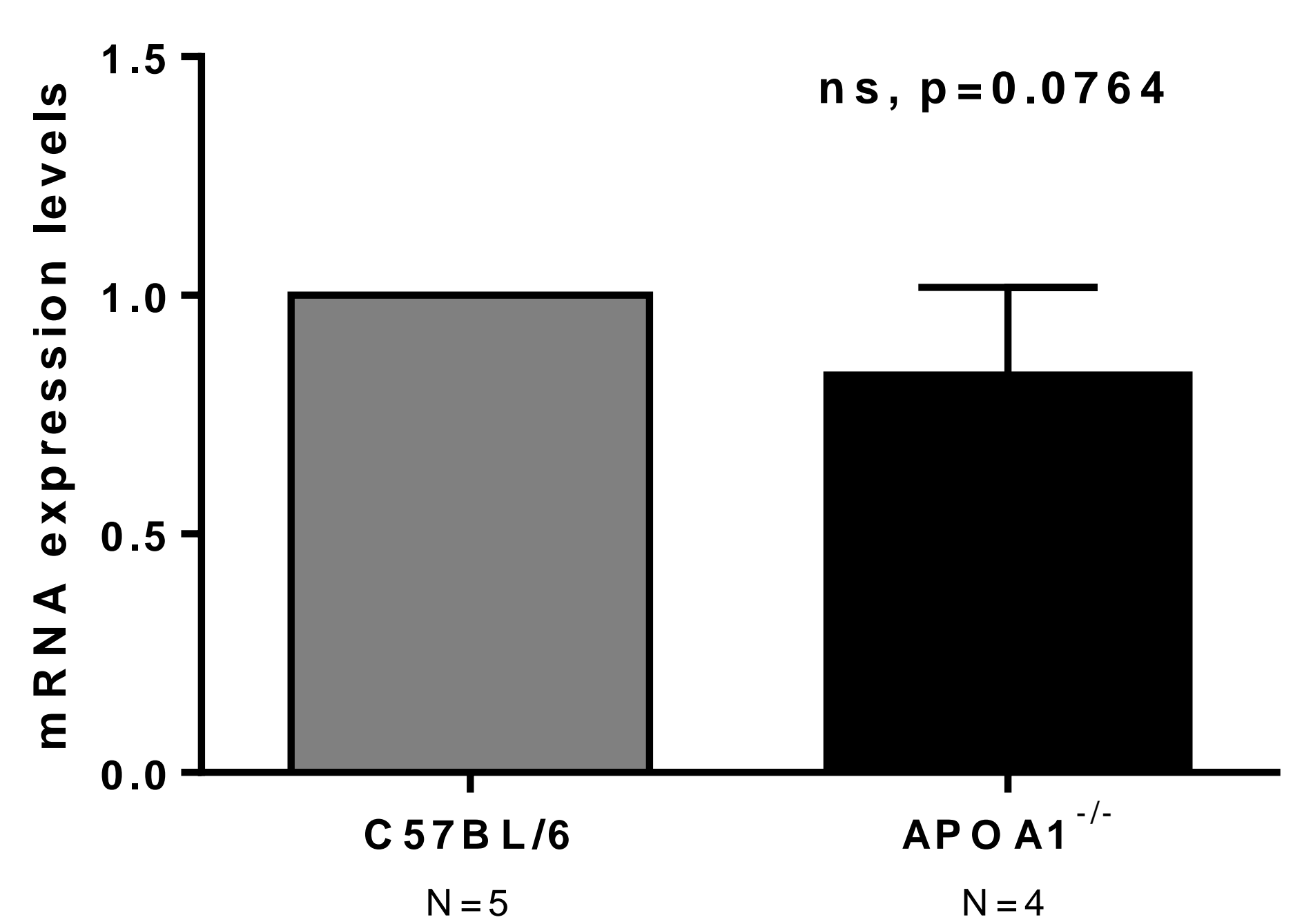

\section{ACKNOWLEDGMENTS}

This study is supported by "The European Community's Seventh Framework Programme (FP7-IR-Grant-PIRG06-GA-256402)" and The University of Patras "Karatheodori" Research Grants \# D.155 \& E.073 and is part of the research network "OsteoNet" of the University of Patras activities.

There is no conflict of interest to declare.

\section{SELECTED BIBLIOGRAPHY}

. Blair et al. A polipoprotein A-1 regulates osteoblast and lipoblast precursor cells in mice.

Lab Invest. 2016; doi: 10.1038/labinvest.2016.51. [Epub ahead of print]

2. Triantaphyllidou et al. Perturbations in the HDL metabolic pathway predispose to the development of osteoarthritis in mice following long-term exposure to western-type diet. Osteoarthritis Cartilage 2013; 21 (2):322-30. doi: 10.1016/j.joca.2012.11.003.

3. Pawig et al. Diversity and inter-connections in the CXCR4 chemokine receptor/ligand family: molecular perspectives. Front Immunol. 2015; 21 (6):429. doi: 10.3389/fimmu.2015.00429.

4. Papachristou and Blair. Bone and high-density lipoprotein: The beginning of a beautiful friendship. World J Orthop. 2016; 7 (2): 74-77. DOI: 10.5312/wjo.v7.i2.74. 